\title{
Metal Coordination and Imine-Amine Hydrogen Bonding as Source of Strongly Shifted Adenine $\mathrm{p} K_{\mathrm{a}}$ Values.
}

Michael Roitzsch and Bernhard Lippert

Fachbereich Chemie, Universität Dortmund, 44221 Dortmund, Germany

Supporting Information: Determination of $p K_{a}$ values by means of $p D$ dependent ${ }^{1}$ H NMR spectroscopy

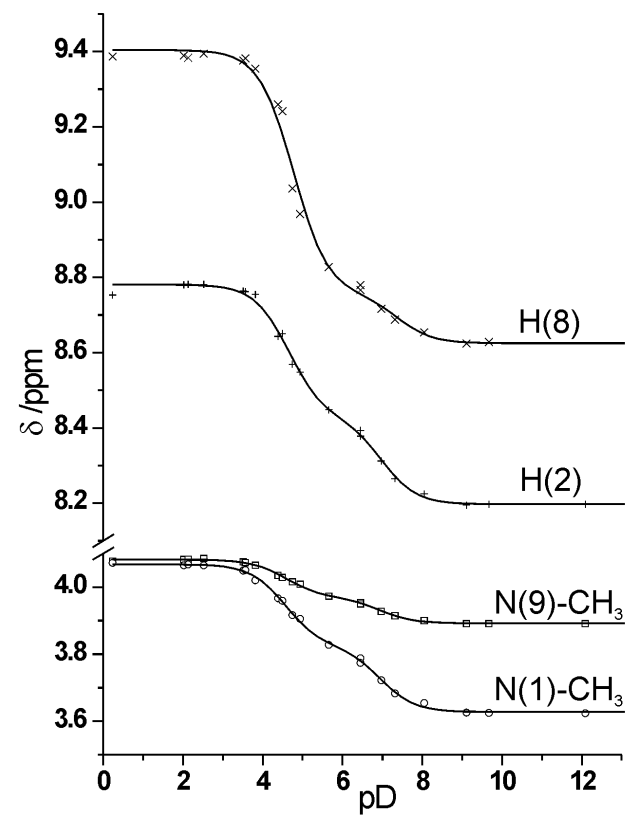

For least-squares fitting the following equation was used ${ }^{1}$

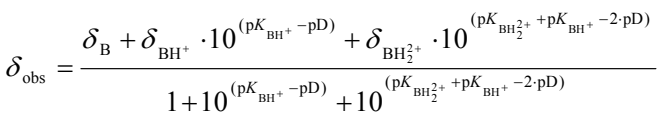

The $\mathrm{p} K_{\mathrm{a}}$ values obtained by this procedure are valid for heavy water. The corresponding $\mathrm{p} K_{\mathrm{a}}$ values for $\mathrm{H}_{2} \mathrm{O}$ were calculated using the following equation ${ }^{2}$ $\mathrm{p} K^{\mathrm{H}_{2} \mathrm{O}}=\frac{\mathrm{p} K^{\mathrm{D}_{2} \mathrm{O}}-0.45}{1.015}$

Figure 1: Plot of the chemical shifts of trans$\left[\left(\mathrm{NH}_{3}\right)_{2} \mathrm{Pt}(1,9-\mathrm{DimeAH}-N 7)_{2}\right]^{4+}$ vs. $\mathrm{pD}$ value.

Tabular 1: Parameters of the non-linear least-squares fits

\begin{tabular}{lcccc}
\hline Parameter & $\mathrm{CH}_{3}$ & $\mathrm{CH}_{3}$ & $\mathrm{H}(8)$ & $\mathrm{H}(2)$ \\
\hline$\delta\left\{\right.$ trans $\left.-\left[\left(\mathrm{NH}_{3}\right)_{2} \mathrm{Pt}(1,9-\mathrm{DimeA}-N 7)_{2}\right]^{2+}\right\}$ & $3.891(2)$ & $3.627(3)$ & $8.62(2)$ & $8.197(7)$ \\
\hline$\delta\left\{\right.$ trans $\left.^{2+}\left[\left(\mathrm{NH}_{3}\right)_{2} \mathrm{Pt}\left(1,9-\mathrm{DimeAH}^{+}-N 7\right)(1,9-\mathrm{DimeA}-N 7)\right]^{3+}\right\}$ & $3.970(3)$ & $3.825(8)$ & $8.75(3)$ & $8.42(2)$ \\
$\delta\left\{\right.$ trans $\left.^{+}\left[\left(\mathrm{NH}_{3}\right)_{2} \mathrm{Pt}\left(1,9-\mathrm{DimeAH}^{+}-N 7\right)\right]^{4+}\right\}$ & $4.082(1)$ & $4.067(3)$ & $9.40(1)$ & $8.780(5)$ \\
$\mathrm{p} K_{\mathrm{a}, 1}{ }_{\mathrm{D} 2 \mathrm{O}}$ & $4.56(4)$ & $4.56(5)$ & $4.76(7)$ & $4.65(6)$ \\
$\mathrm{p} K_{\mathrm{a}, 2}{ }_{\mathrm{D} 2 \mathrm{O}}$ & $6.92(6)$ & $6.94(6)$ & $7.4(5)$ & $6.9(1)$ \\
$\mathrm{p} K_{\mathrm{a}, 1}{ }_{\mathrm{H} 2 \mathrm{O}}$ & $4.05(4)$ & $4.05(3)$ & $4.25(7)$ & $4.14(6)$ \\
$\mathrm{p} K_{\mathrm{a}, 2}{ }^{2}$ & $6.37(6)$ & $6.39(6)$ & $6.9(5)$ & $6.4(1)$ \\
\hline
\end{tabular}

\section{References}

${ }^{1}$ Tribolet, R.; Sigel, H. Eur. J. Biochem. 1987, 163, 353-363.

${ }^{2}$ Martin, R. B. Science 1963, 139, 1198-1203. 